\title{
NONLINEAR OBSERVER TECHNIQUES FOR OSCILLATORY FAILURE CASE DETECTION
}

\section{Efimov ${ }^{1}$ and A. Zolghadri ${ }^{2}$}

${ }^{1}$ INRIA - LNE

Parc Scientifique de la Haute Borne 40 Avenue Halley, Bât. A Park Plaza, Villeneuve d'Ascq 59650, France

${ }^{2}$ University of Bordeaux, IMS-lab, Automatic Control Group 351 Cours de la Libération, Talence 33405, France

\begin{abstract}
The problem of observer design for fault detection in a class of nonlinear systems subject to parametric and signal uncertainties is studied. The design procedure includes formalized optimization of observer free parameters in terms of trade-offs for fault detection performance and robustness to external disturbances or model uncertainties. The technique makes use of some monotonicity conditions imposed on the estimation error dynamics. Efficiency of the proposed approach is demonstrated through the Oscillatory Failure Case (OFC) in aircraft control surface servoloops.
\end{abstract}

\section{INTRODUCTION}

Model-based Fault Detection and Isolation (FDI) in dynamical systems is an active research area (for a recent survey, see $[1,2]$ ). An important focus has been made on the use of observer-based schemes. In the linear case, it has been shown that any linear fault detection filter can be transformed into an observer-based form [3], providing a unified framework for analysis and implementation [4-8]. From an estimation point of view, the problem of optimal noise filtering for stochastic linear systems has many solutions [9,10]. For nonlinear systems, a general framework does not exist, although numerical or suboptimal solutions are available [11-13]. Typically, the observer design problem is solvable for a canonical representation of nonlinear systems $[14,15]$.

In this paper, an approach is developed for nonlinear fault detection observer design, together with a procedure for parameter tuning. For the latter, the design is made under monotonicity assumption [16] for the estimation error dynamic. 
In this case, using an appropriate linear parameter varying (LPV) transformation [17-19], the design of minorant and majorant monotone linear systems is possible, whose solutions create an envelope for the original system trajectories. Solving an optimization problem for the minorant and majorant systems (the solution is straightforward due to their linearity), it is possible to obtain a suboptimal solution for the original nonlinear system (a local optimality is ensured when the system solutions converge to the minorant or majorant flows). The goal is to maximize robustness with respect to disturbances and sensitivity with respect to faults.

In some cases, the faulty signal is known to belong to a specific class of signals (e.g., the harmonic functions of time with predefined frequency range). For example, in the ODC detection considered in [20], the faults are assumed to be harmonic. Such a priori available information simplifies searched solution, since specified techniques oriented on analysis of the harmonic input response can be used for design of observer gains.

The following nonlinear system is considered:

$$
\dot{\mathbf{x}}=\mathbf{A x}+\mathbf{G F}(\mathbf{H x}, \mathbf{u}+\mathbf{f}, \theta)+\mathbf{S v} ; \mathbf{y}=\mathbf{C x}+\mathbf{d}
$$

where $\mathbf{x} \in \mathbb{R}^{n}, \mathbf{u} \in \mathbb{R}^{m}$, and $\mathbf{y} \in \mathbb{R}^{p}$ are the system state, input, and output; $\mathbf{v} \in \mathbb{R}^{v}$ and $\mathbf{d} \in \mathbb{R}^{p}$ are the state and the output disturbances; $\mathbf{f} \in \mathbb{R}^{m}$ is the faulty signal (unknown portion of the input); $\theta \in \mathbb{R}^{q}$ is the vector of unknown parameters; the matrices $\mathbf{A}, \mathbf{G}, \mathbf{H}, \mathbf{S}$, and $\mathbf{C}$ are known and constant having appropriate dimensions; and the function $\mathbf{F}: \mathbb{R}^{l+m+q} \rightarrow \mathbb{R}^{g}$ is continuously differentiable. The matrices $\mathbf{G}$ and $\mathbf{H}$ are introduced to take into account more accurately the influence of nonlinearity on the system behavior. For simplicity of presentation, the signal $\mathbf{f}$ is considered to act on the control signal as additive disturbance. Such a restriction is motivated by the numerical example study from aeronautic field in section 6 . However, the approach can be applied to multiplicative faults also. Assume that all input signals $\mathbf{u}, \mathbf{f}, \mathbf{v}$, and $\mathbf{d}$ are (Lebesgue) measurable and essentially bounded, i. e.,

$$
\|\mathbf{f}\| \text { ess } \sup _{t \geq 0}|\mathbf{f}(t)|<+\infty .
$$

The objective is to design an observer for (1) using the available noisy measurements $\mathbf{y}$ and the input $\mathbf{u}$, and ensuring robustness with respect to the uncertain parameters $\theta$ and the signals $\mathbf{v}$ and $\mathbf{d}$. Moreover, for fault detection, it is required to find the observer gains maximizing sensitivity of the output estimation error with respect to $\mathbf{f}$ and robustness with respect to $\mathbf{v}$ and $\mathbf{d}$. Note that if the fault detection problem is not of interest, then $\mathbf{f}$ can be considered as an additional unknown input.

Two solutions of this problem are presented below. One is more conventional and it is based on LMIs verification (see section 4). It is shown that optimization 
in this framework is complicated. Another solution is the main contribution of the work, and it utilizes the monotone system routine for analysis and optimization (see section 5).

The paper is organized as follows. Preliminaries are given in section 2. The observer equations are introduced in section 3. Stability conditions based on LMIs are presented in section 4 (the optimization possibilities of this approach are also discussed). An alternative approach (monotone system theory) for the observer stability analysis and the new optimization technique are given in section 5. In section 6 , the overall approach is illustrated through its application to OFC detection in aircraft control surface servoloops.

\section{PRELIMINARIES}

This section introduces some basic notions about monotone systems and LPV representation of nonlinearities.

\subsection{Linear Parameter Varying Representation of Nonlinear Functions}

For any two vectors $\mathbf{p}$ and $\mathbf{p}^{\prime}$ of the same dimension, let define

$$
L\left(\mathbf{p}, \mathbf{p}^{\prime}\right)=\left\{\lambda \mathbf{p}+(1-\lambda) \mathbf{p}^{\prime}, \quad 0 \leq \lambda \leq 1\right\}
$$

(the line connecting the points $\mathbf{p}$ and $\mathbf{p}^{\prime}$ ). Since the vector valued function $\mathbf{F}$ in (1) is continuously differentiable, then according to the Mean Value Theorem, for any $\mathbf{h}, \mathbf{h}^{\prime} \in \mathbf{H} \mathcal{X}, \mathbf{u}, \mathbf{u}^{\prime} \in \mathcal{U} \cup \mathcal{F}, \boldsymbol{\theta}, \boldsymbol{\theta}^{\prime} \in \boldsymbol{\Omega}$, there exist $\eta_{j}^{h} \in L\left(\mathbf{h}, \mathbf{h}^{\prime}\right), \eta_{j}^{u}$ $\in L\left(\mathbf{u}, \mathbf{u}^{\prime}\right), \eta_{j}^{\theta} \in L\left(\boldsymbol{\theta}, \boldsymbol{\theta}^{\prime}\right), j=\overline{1, g}$ such that

$$
\begin{gathered}
\mathbf{F}(\mathbf{h}, \mathbf{u}, \boldsymbol{\theta})-\mathbf{F}\left(\mathbf{h}^{\prime}, \mathbf{u}^{\prime}, \boldsymbol{\theta}^{\prime}\right)=\Delta_{x}\left(\mathbf{h}-\mathbf{h}^{\prime}\right)+\Delta_{u}\left(\mathbf{u}-\mathbf{u}^{\prime}\right)+\Delta_{\theta}\left(\boldsymbol{\theta}-\boldsymbol{\theta}^{\prime}\right) \\
\Delta_{x, j}=\left.\frac{\partial F_{j}(\boldsymbol{\xi}, \mathbf{v}, \mathbf{q})}{\partial \boldsymbol{\xi}}\right|_{\boldsymbol{\xi}=\boldsymbol{\eta}_{j}^{x}, \mathbf{v}=\boldsymbol{\eta}_{j}^{u}, \mathbf{q}=\boldsymbol{\eta}_{j}^{\theta}} ; \Delta_{u, j}=\left.\frac{\partial F_{j}(\boldsymbol{\xi}, \mathbf{v}, \mathbf{q})}{\partial \mathbf{v}}\right|_{\boldsymbol{\xi}=\boldsymbol{\eta}_{j}^{x}, \mathbf{v}=\boldsymbol{\eta}_{j}^{u}, \mathbf{q}=\boldsymbol{\eta}_{j}^{\theta}} \\
\Delta_{\theta, j}=\left.\frac{\partial \mathbf{F}(\boldsymbol{\xi}, \mathbf{v}, \mathbf{q})}{\partial \mathbf{q}}\right|_{\boldsymbol{\xi}=\boldsymbol{\eta}_{j}^{x}, \mathbf{v}=\boldsymbol{\eta}_{j}^{u}, \mathbf{q}=\boldsymbol{\eta}_{j}^{\theta}}, j=\overline{1, g}
\end{gathered}
$$

where the symbols $\Delta_{x, j}, \Delta_{u, j}, \Delta_{\theta, j}$ denote the $j$ th row of the corresponding matrix.

The application of this technique gives an exact equivalent LPV representation of a nonlinear function. It is not a linearization around a single point (or around a trajectory) since the above expression is an equality. The LPV 
approach allows to transform nonlinear models to the linear ones depending on unknown parameters $\Delta_{x}, \Delta_{u}$, and $\Delta_{\theta}$. Therefore, the complexity of the nonlinear model (1) can be replaced with enlarged parametric uncertainty of a linear one. This tool will be applied in the next section to analyze the estimation error dynamics of the observer.

\subsection{Monotone System Theory}

The system

$$
\dot{\mathbf{x}}=\mathbf{f}(t, \mathbf{x}), \quad \mathbf{x} \in X, t \geq 0,
$$

with the solution $\mathbf{x}\left(t, \mathbf{x}_{0}\right)$ for the initial condition $\mathbf{x}(0)=\mathbf{x}_{0}$ is called monotone, if $\mathbf{x}_{0} \leq \boldsymbol{\xi}_{0} \Rightarrow \mathbf{x}\left(t, \mathbf{x}_{0}\right) \leq \mathbf{x}\left(t, \boldsymbol{\xi}_{0}\right)$ for all $t \geq 0$ [16] (for the vectors $\mathbf{x}_{0}$ and $\boldsymbol{\xi}_{0}$, the inequality $\mathbf{x}_{0} \leq \boldsymbol{\xi}_{0}$ is understood elementwise). The system is called cooperative if $\partial f_{i}(t, \mathbf{x}) / \partial x_{j} \geq 0$ for all $1 \leq i \neq j \leq n, t \in R$ and $\mathbf{x} \in X$ [16]. Cooperative systems form a subclass of monotone ones. A matrix $\mathbf{A}$ with dimension $n \times n$ is called Metzler if $A_{i, j} \geq 0$ for all $1 \leq i \neq j \leq n$. Note that for the cooperative stable system (the matrix $\mathbf{A}$ is Metzler and Hurwitz),

$$
\dot{\mathbf{s}}(t)=\mathbf{A} \mathbf{s}(t)+\mathbf{r}(t), \quad \mathbf{s} \in R^{n}, \mathbf{r} \in R^{n}, \quad t \leq 0,
$$

the properties $\mathbf{s}(0) \geq 0, \mathbf{r}(t) \geq 0$ for all $t \geq 0$ imply $\mathbf{s}(t) \geq 0$ for $t \geq 0$ and, conversely, $\mathbf{s}(0) \leq 0, \mathbf{r}(t) \leq 0$ for all $t \geq 0$ ensures $\mathbf{s}(t) \leq 0$ for $t \geq 0$. The system is called competitive if $\partial f_{i}(t, \mathbf{x}) / \partial x_{j} \leq 0$ for all $1 \leq i \neq j \leq n, t \in R$ and $\mathbf{x} \in X$; in backward time, the competitive systems behave like the cooperative ones [16].

\section{ROBUST OBSERVER EQUATIONS}

This section is based on the following assumption.

Assumption 1. Let the compact sets $\mathcal{X} \subset \mathbb{R}^{n}, \mathcal{U} \subset \mathbb{R}^{m}, \mathcal{F} \subset \mathbb{R}^{m}, \mathcal{V} \subset \mathbb{R}^{v}$, $\mathcal{D} \subset \mathbb{R}^{p}$, and $\boldsymbol{\Omega} \subset \mathbb{R}^{q}$ be given such that for almost all $t \geq 0$,

$$
\mathbf{x}(t) \in \mathcal{X} ; \mathbf{u}(t) \in \mathcal{U} ; \mathbf{f}(t) \in \mathcal{F} ; \mathbf{v}(t) \in \mathcal{V} ; \mathbf{d}(t) \in \mathcal{D} ; \boldsymbol{\theta} \in \boldsymbol{\Omega} .
$$

Such constraints are rather common in nonlinear observer design theory stating that the system (1) has bounded inputs and the state with some known upper bounds.

Consider the following Luenberger type observer for (1):

$$
\begin{aligned}
& \dot{\mathbf{z}}=\mathbf{Z}(\mathbf{z}, \mathbf{y}, \mathbf{u})=\mathbf{A z} \\
+ & \mathbf{G F}\left[\mathbf{H z}+\mathbf{L}_{2}(\mathbf{y}-\mathbf{C z}), \mathbf{u}+\mathbf{L}_{3}(\mathbf{y}-\mathbf{C z}), \boldsymbol{\theta}^{*}+\mathbf{L}_{4}(\mathbf{y}-\mathbf{C z})\right]+\mathbf{L}_{1}(\mathbf{y}-\mathbf{C z})
\end{aligned}
$$


where $\mathbf{z} \in \mathbb{R}^{n}$ is the estimate of the state $\mathbf{x} ; \mathbf{L}_{i}, i=\overline{1,4}$, are the observer gains to be designed; and $\boldsymbol{\theta}^{*} \in \boldsymbol{\Omega}$ is a supporting fixed value for the vector of unknown parameters. In (2), the output injection term is introduced for all arguments of the nonlinear function $\mathbf{F}$. The gain $\mathbf{L}_{1}$ is standard, it is used to ensure stability of the pure linear part of the estimation error $\mathbf{e}=\mathbf{x}-\mathbf{z}$ dynamics. The gain $\mathbf{L}_{2}$ has been proposed in [21] in order to improve the robustness abilities of (2) and to relax restrictiveness of the LMIs used for the observer design. The gains $\mathbf{L}_{3}$ and $\mathbf{L}_{4}$ have been introduced in [22] to improve robustness of the system with respect to $\mathbf{v}, \mathbf{d}, \boldsymbol{\theta}$ and sensitivity with respect to $\mathbf{f}$. These gains have to be assigned to guarantee (or to find a trade-off) the system stability and performance, and to satisfy the required estimation and fault detection specifications.

To apply the LPV technique below, the observer (2) has to be equipped with a projection algorithm ensuring that $\mathbf{z}(t)$ belongs to the set $\mathcal{X}$ for all $t \geq 0$ :

$$
\dot{\mathbf{z}}=\underset{\mathcal{X}}{\operatorname{proj}}\{\mathbf{Z}(\mathbf{z}, \mathbf{y}, \mathbf{u})\}
$$

the equations of the projection algorithm can be found in [23] (smooth projection).

From (1) and (2), the estimation error -+e dynamics can be given by:

$$
\begin{aligned}
& \dot{\mathbf{e}}=\mathbf{A x}+\mathbf{G F}(\mathbf{H} \mathbf{x}, \mathbf{u}+\mathbf{f}, \boldsymbol{\theta})+\mathbf{S v}-\mathbf{A z} \\
& -\mathbf{G F}\left[\mathbf{H z}+\mathbf{L}_{2}(\mathbf{y}-\mathbf{C z}), \mathbf{u}+\mathbf{L}_{3}(\mathbf{y}-\mathbf{C z}), \boldsymbol{\theta}^{*}+\mathbf{L}_{4}(\mathbf{y}-\mathbf{C z})\right]-\mathbf{L}_{1}(\mathbf{y}-\mathbf{C z}) \\
& \quad=\left(\mathbf{A}-\mathbf{L}_{1} \mathbf{C}\right) \mathbf{e}-\mathbf{L}_{1} \mathbf{d}+\mathbf{S v} \\
& +\mathbf{G}\left\{\mathbf{F}(\mathbf{H} \mathbf{x}, \mathbf{u}+\mathbf{f}, \boldsymbol{\theta})-\mathbf{F}\left[\mathbf{H z}+\mathbf{L}_{2}(\mathbf{y}-\mathbf{C z}), \mathbf{u}+\mathbf{L}_{3}(\mathbf{y}-\mathbf{C z}), \boldsymbol{\theta}^{*}+\mathbf{L}_{4}(\mathbf{y}-\mathbf{C z})\right]\right\}
\end{aligned}
$$

Under Assumption 1 with $\mathbf{z} \in \mathcal{X}$ due to (3) and applying the LPV transformation method, it can be shown that there exist some maps $\Delta_{x}: \mathbb{R} \rightarrow \mathbb{R}^{g \times l}$, $\Delta_{u}: \mathbb{R} \rightarrow \mathbb{R}^{g \times m}$, and $\Delta_{\theta}: \mathbb{R} \rightarrow \mathbb{R}^{g \times q}$ such that for all $t \geq 0$,

$$
\begin{aligned}
\mathbf{F}(\mathbf{H x}, \mathbf{u}+\mathbf{f}, \boldsymbol{\theta})-\mathbf{F}\left[\mathbf{H z}+\mathbf{L}_{2}(\mathbf{y}-\mathbf{C z}), \mathbf{u}+\right. & \left.\mathbf{L}_{3}(\mathbf{y}-\mathbf{C z}), \boldsymbol{\theta}^{*}+\mathbf{L}_{4}(\mathbf{y}-\mathbf{C z})\right] \\
=\Delta_{z}(t)\left[\left(\mathbf{H}-\mathbf{L}_{2} \mathbf{C}\right) \mathbf{e}-\mathbf{L}_{2} \mathbf{d}\right]+ & \delta_{u}(t)\left[\mathbf{f}-\mathbf{L}_{3} \mathbf{C e}-\mathbf{L}_{3} \mathbf{d}\right] \\
& +\Delta_{\theta}(t)\left[\boldsymbol{\theta}-\boldsymbol{\theta}^{*}-\mathbf{L}_{4} \mathbf{C e}-\mathbf{L}_{4} \mathbf{d}\right]
\end{aligned}
$$

The exact values of the matrix functions $\Delta_{x}(t), \Delta_{u}(t)$, and $\Delta_{\theta}(t)$ are unknown, but the set of admissible values is known (the values of the function $\mathbf{F}$ gradient on $\mathcal{X}, \mathcal{U}, \mathcal{F}, \mathcal{V}, \mathcal{D}$, and $\Omega \subset \mathbb{R}^{q}$ ), i.e., there are the known sets of matrices $\Upsilon_{x}, \Upsilon_{u}$, and $\Upsilon_{\theta}$ such that $\Delta_{x}(t) \in \Upsilon_{x}, \Delta_{u}(t) \in \Upsilon_{u}, \Delta_{\theta}(t) \in \Upsilon Y_{\theta}$ for all $t \geq 0$.

Having enabled the projection algorithm (3), an LPV transformation can be applied to the equation of estimation error dynamics: 


$$
\begin{aligned}
& \dot{\mathbf{e}}=\left\{\left(\mathbf{A}-\mathbf{L}_{1} \mathbf{C}\right)+\mathbf{G}\left[\Delta_{x}(t)\left(\mathbf{H}-\mathbf{L}_{2} \mathbf{C}\right)-\Delta_{u}(t) \mathbf{L}_{3} \mathbf{C}-\Delta_{\theta}(t) \mathbf{L}_{4} \mathbf{C}\right]\right\} \mathbf{e} \\
&-\left\{\mathbf{L}_{1}+\mathbf{G}\left[\Delta_{x}(t) \mathbf{L}_{2}\right.\right.\left.\left.+\Delta_{u}(t) \mathbf{L}_{3}+\Delta_{\theta}(t) \mathbf{L}_{4}\right]\right\} \mathbf{d} \\
&+\mathbf{S v}+\mathbf{G}\left\{\Delta_{u}(t) \mathbf{f}+\Delta_{\theta}(t)\left[\boldsymbol{\theta}-\boldsymbol{\theta}^{*}\right]\right\} .
\end{aligned}
$$

Remark. As it can be concluded from (4), the influence of the measurement noise $\mathbf{d}$ is hard to attenuate since the multiplicative gain for this input is proportional to the sum of all $\mathbf{L}_{i}, i=\overline{1,4}$. However, robustness with respect to the inputs $\mathbf{v}$ and $\mathbf{f}$ and the parametric mismatch $\boldsymbol{\theta}-\boldsymbol{\theta}^{*}$ can be augmented by a proper choice of the gains $\mathbf{L}_{i}$, $i=\overline{1,4}$ (the same with the sensitivity with respect to $\mathbf{f}$ ).

In the following sections, two techniques are presented for stability analysis of (4) and performance improvement in (2) and (3) as well.

\section{STABILITY CONDITIONS BASED ON LINEAR MATRIX INEQUALITIES}

Denote the identity matrix with dimension $n \times n$ by $\mathbf{I}_{n}$ and state the symbols $\lambda_{\max }(\mathbf{P})$ and $\lambda_{\min }(\mathbf{P})$ for the maximal and minimal eigenvalues of a square ma$\operatorname{trix} \mathbf{P}$.

\subsection{Stability Conditions}

Theorem 1. Assume that

(1) assumption 1 is satisfied;

(2) there exist matrices $\mathbf{W}_{x}, \mathbf{W}_{u}$, and $\mathbf{W}_{\theta}$ such that

$$
\begin{gathered}
\tau_{x} \Delta_{x}^{\mathrm{T}} \Delta_{x}-\Delta_{x}^{\mathrm{T}} \mathbf{W}_{x}-\mathbf{W}_{x}^{\mathrm{T}} \Delta_{x}-\alpha_{x} \mathbf{I}_{g} \leq 0 ; \\
\tau_{u} \Delta_{u}^{\mathrm{T}} \Delta_{u}-\Delta_{u}^{\mathrm{T}} \mathbf{W}_{u}-\mathbf{W}_{u}^{\mathrm{T}} \Delta_{u}-\alpha_{u} \mathbf{I}_{g} \leq 0 \\
\tau_{\theta} \Delta_{\theta}^{\mathrm{T}} \Delta_{\theta}-\Delta_{\theta}^{\mathrm{T}} \mathbf{W}_{\theta}-\mathbf{W}_{\theta}^{\mathrm{T}} \Delta_{\theta}-\alpha_{\theta} \mathbf{I}_{g} \leq 0
\end{gathered}
$$

for all $\Delta_{x} \in \Upsilon_{x}, \Delta_{u} \in \Upsilon_{u}$, and $\Delta_{\theta} \in \Upsilon_{\theta}$ and some real $\tau_{x}, \tau_{y}, \tau_{\theta}, \alpha_{x}, \alpha_{u}$, and $\alpha_{\theta} ;$ and

(3) the gains $\mathbf{L}_{i}, i=\overline{1,4}$ for some $\mathbf{P}=\mathbf{P}^{T}>0$ admit the matrix inequality:

$$
\mathbf{Y}=\left[\begin{array}{ccccc}
\left(\mathbf{A}-\mathbf{L}_{1} \mathbf{C}\right)^{\mathrm{T}} \mathbf{P}+\mathbf{P}\left(\mathbf{A}-\mathbf{L}_{1} \mathbf{C}\right) & \mathbf{P G}-\mathbf{P G}-\mathbf{P G} \\
\mathbf{G}^{\mathrm{T}} \mathbf{P} & 0 & 0 & 0 \\
-\mathbf{G}^{\mathrm{T}} \mathbf{P} & 0 & 0 & 0 \\
-\mathbf{G}^{\mathrm{T}} \mathbf{P} & 0 & 0 & 0
\end{array}\right]
$$




$$
\leq\left[\begin{array}{cccc}
\mathbf{Z} & -\left(\mathbf{H}-\mathbf{L}_{2} \mathbf{C}\right)^{\mathrm{T}} \mathbf{W}_{x}^{\mathrm{T}}-\left(\mathbf{L}_{3} \mathbf{C}\right)^{\mathrm{T}} \mathbf{W}_{u}^{\mathrm{T}}-\left(\mathbf{L}_{4} \mathbf{C}\right)^{\mathrm{T}} \mathbf{W}_{\theta}^{\mathrm{T}} \\
-\mathbf{W}_{x}\left(\mathbf{H}-\mathbf{L}_{2} \mathbf{C}\right) & \tau_{x} \mathbf{I}_{g} & 0 & 0 \\
-\mathbf{W}_{u} \mathbf{L}_{3} \mathbf{C} & 0 & \tau_{u} \mathbf{I}_{g} & 0 \\
-\mathbf{W}_{\theta} \mathbf{L}_{4} \mathbf{C} & 0 & 0 & \tau_{\theta} \mathbf{I}_{g}
\end{array}\right]
$$

where

$$
\mathbf{Z}=-\nu \mathbf{I}_{n}-\alpha_{x}\left(\mathbf{H}-\mathbf{L}_{2} \mathbf{C}\right)^{\mathrm{T}}\left(\mathbf{H}-\mathbf{L}_{2} \mathbf{C}\right)-\alpha_{u}\left(\mathbf{L}_{3} \mathbf{C}\right)^{\mathrm{T}}\left(\mathbf{L}_{3} \mathbf{C}\right)-\alpha_{\theta}\left(\mathbf{L}_{4} \mathbf{C}\right)^{\mathrm{T}}\left(\mathbf{L}_{4} \mathbf{C}\right) .
$$

Then, in (1)-(3) for all $t \geq 0$, one has:

$$
\begin{aligned}
|\mathbf{e}(t)| \leq \kappa\{|\mathbf{e}(0)| & e^{-0.25 \nu \lambda_{\max }^{-1}(\mathbf{P}) t} \\
& \left.+4 \nu^{-1}\left(\sqrt{\lambda_{d}}\|\mathbf{d}\|+\sqrt{\lambda_{s}}\|\mathbf{s}\|+\sqrt{\lambda_{f}}\|\mathbf{f}\|+\sqrt{\lambda_{\theta}}\left|\boldsymbol{\theta}-\boldsymbol{\theta}^{*}\right|\right)\right\}
\end{aligned}
$$

where

$$
\begin{gathered}
\lambda_{s}=\lambda_{\max }\left(\mathbf{S}^{\mathrm{T}} \mathbf{P P S}\right) ; \lambda_{f}=\max _{\Delta_{u} \in \Upsilon_{u}} \lambda_{\max }\left(\Delta_{u}^{\mathrm{T}} \mathbf{G}^{\mathrm{T}} \mathbf{P} \mathbf{P G} \Delta_{u}\right) ; \\
\lambda_{d}=\max _{\Delta_{x} \in \Upsilon_{x}, \Delta_{u} \in \Upsilon_{u}, \Delta_{\theta} \in \Upsilon_{\theta}}\left(\{ \mathbf { L } _ { 1 } + \mathbf { G } [ \Delta _ { x } \mathbf { L } _ { 2 } + \Delta _ { u } \mathbf { L } _ { 3 } + \Delta _ { \theta } \mathbf { L } _ { 4 } ] \} ^ { \mathrm { T } } \mathbf { P P } \left\{\mathbf{L}_{1}\right.\right. \\
\left.\left.+\mathbf{G}\left[\Delta_{x} \mathbf{L}_{2}+\Delta_{u} \mathbf{L}_{3}+\Delta_{\theta} \mathbf{L}_{4}\right]\right\}\right) ; \\
\kappa=\sqrt{\frac{\lambda_{\max }(\mathbf{P})}{\lambda_{\min }(\mathbf{P})} ; \lambda_{\theta}=\max _{\Delta_{\theta} \in \Upsilon_{\theta}} \lambda_{\max }\left(\Delta_{\theta}^{\mathrm{T}} \mathbf{G}^{\mathrm{T}} \mathbf{P P G} \Delta_{\theta}\right) .}
\end{gathered}
$$

Proof. The projection algorithm ensures the trajectories boundedness in the large $(\mathbf{z}(t) \in \mathcal{X}$ for all $t \geq 0)$. Let analyze the error dynamics (4) into the set $\mathcal{X}$ using the Lyapunov function $\mathbf{V}(\mathbf{e})=\mathbf{e}^{\mathrm{T}} \mathbf{P e}$ :

$$
\begin{aligned}
& \dot{V}=\mathbf{e}^{\mathrm{T}}\left[\left(\mathbf{A}-\mathbf{L}_{1} \mathbf{C}\right)^{\mathrm{T}} \mathbf{P}+\right.\left.\mathbf{P}\left(\mathbf{A}-\mathbf{L}_{1} \mathbf{C}\right)\right] \mathbf{e} \\
&+2 \mathbf{e}^{\mathrm{T}} \mathbf{P}\left\{\mathbf{G}\left[\Delta_{x}(t)\left(\mathbf{H}-\mathbf{L}_{2} \mathbf{C}\right)-\Delta_{u}(t) \mathbf{L}_{3} \mathbf{C}-\Delta_{\theta}(t) \mathbf{L}_{4} \mathbf{C}\right]\right\} \mathbf{e} \\
&-2 \mathbf{e}^{\mathrm{T}} \mathbf{P}\left\{\mathbf{L}_{1}+\mathbf{G}\left[\Delta_{x}(t) \mathbf{L}_{2}+\Delta_{u}(t) \mathbf{L}_{3}+\Delta_{\theta}(t) \mathbf{L}_{4}\right]\right\} \mathbf{d} \\
&+2 \mathbf{e}^{\mathrm{T}} \mathbf{P S v}+2 \mathbf{e}^{\mathrm{T}} \mathbf{P} \mathbf{G}\left\{\Delta_{u}(t) \mathbf{f}+\Delta_{\theta}(t)\left[\boldsymbol{\theta}-\boldsymbol{\theta}^{*}\right]\right\} .
\end{aligned}
$$

For $\rho(t, \mathbf{e})=\left[\mathbf{e} \Delta_{x}(t)\left(\mathbf{H}-\mathbf{L}_{2} \mathbf{C}\right) \mathbf{e} \Delta_{u}(t) \mathbf{L}_{3} \mathbf{C e} \Delta_{\theta}(t) \mathbf{L}_{4} \mathbf{C e}\right]^{\mathrm{T}}$, applying some algebra, one gets:

$$
\begin{aligned}
\dot{V}=\rho(t, \mathbf{e})^{\mathrm{T}} \mathbf{Y} \boldsymbol{\rho}(t, \mathbf{e})-2 \mathbf{e}^{\mathrm{T}} \mathbf{P}\left\{\mathbf{L}_{1}+\mathbf{G}\left[\Delta_{x}(t) \mathbf{L}_{2}+\Delta_{u}(t) \mathbf{L}_{3}+\Delta_{\theta}(t) \mathbf{L}_{4}\right]\right\} \mathbf{d} \\
+2 \mathbf{e}^{\mathrm{T}} \mathbf{P S} \mathbf{v}+2 \mathbf{e}^{\mathrm{T}} \mathbf{P G}\left\{\Delta_{u}(t) \mathbf{f}+\Delta_{u \theta}(t)\left[\boldsymbol{\theta}-\boldsymbol{\theta}^{*}\right]\right\} .
\end{aligned}
$$


Using the matrix inequality for $\mathbf{Y}$ introduced in the theorem, one obtains:

$$
\begin{array}{r}
\rho(t, \mathbf{e})^{\mathrm{T}} \mathbf{Y} \rho(t, \mathbf{e}) \leq \mathbf{e}^{\mathrm{T}}\left\{-\nu \mathbf{I}_{n}+\left(\mathbf{H}-\mathbf{L}_{2} \mathbf{C}\right)^{\mathrm{T}}\left[\tau_{x} \Delta_{x}^{\mathrm{T}}(t) \Delta_{x}(t)-\Delta_{x}^{\mathrm{T}}(t) \mathbf{W}_{x}\right.\right. \\
\left.-\mathbf{W}_{x}^{\mathrm{T}} \Delta_{x}(t)-\alpha_{x} \mathbf{I}_{g}\right]\left(\mathbf{H}-\mathbf{L}_{2} \mathbf{C}\right)+\left(\mathbf{L}_{3} \mathbf{C}\right)^{\mathrm{T}}\left[\tau_{u} \Delta_{u}^{\mathrm{T}}(t) \Delta_{u}(t)\right. \\
\left.-\Delta_{u}^{\mathrm{T}}(t) \mathbf{W}_{u}-\mathbf{W}_{u}^{\mathrm{T}} \Delta_{u}(t)-\alpha_{u} \mathbf{I}_{g}\right]\left(\mathbf{L}_{3} \mathbf{C}\right)+\left(\mathbf{L}_{4} \mathbf{C}\right)^{\mathrm{T}}\left[\tau_{\theta} \Delta_{\theta}^{\mathrm{T}}(t) \Delta_{\theta}(t)\right. \\
\left.\left.-\Delta_{\theta}^{\mathrm{T}}(t) \mathbf{W}_{\theta}-\mathbf{W}_{\theta}^{\mathrm{T}} \Delta_{\theta}(t)-\alpha_{\theta} \mathbf{I}_{g}\right]\left(\mathbf{L}_{4} \mathbf{C}\right)\right\} \mathbf{e} .
\end{array}
$$

Substitution of the inequalities for $\mathbf{W}_{x}, \mathbf{W}_{u}$, and $\mathbf{W}_{\theta}$ gives

$$
\rho(t, \mathbf{e})^{\mathrm{T}} \mathbf{Y} \boldsymbol{\rho}(t, \mathbf{e}) \leq-\nu \mathbf{e}^{\mathrm{T}} \mathbf{e},
$$

then

$$
\begin{aligned}
& \dot{V} \leq-\boldsymbol{\nu} \mathbf{e}^{\mathrm{T}} \mathbf{e}-2 \mathbf{e}^{\mathrm{T}} \mathbf{P}\left\{\mathbf{L}_{1}+\mathbf{G}\left[\Delta_{x}(t) \mathbf{L}_{2}+\Delta_{u}(t) \mathbf{L}_{3}+\Delta_{\theta}(t) \mathbf{L}_{4}\right]\right\} \mathbf{d} \\
&+2 \mathbf{e}^{\mathrm{T}} \mathbf{P S v}+2 \mathbf{e}^{\mathrm{T}} \mathbf{P G}\left\{\Delta_{u}(t) \mathbf{f}+\Delta_{\theta}(t)\left[\boldsymbol{\theta}-\boldsymbol{\theta}^{*}\right]\right\} \\
& \leq-0.5 \boldsymbol{\nu} \mathbf{e}^{\mathrm{T}} \mathbf{e}+8 \boldsymbol{\nu}^{-1}\left(\lambda_{d} \mathbf{d}^{\mathrm{T}} \mathbf{d}+\lambda_{s} \mathbf{s}^{\mathrm{T}} \mathbf{s}+\lambda_{f} \mathbf{f}^{\mathrm{T}} \mathbf{f}+\lambda_{\theta}\left[\boldsymbol{\theta}-\boldsymbol{\theta}^{*}\right]^{\mathrm{T}}\left[\boldsymbol{\theta}-\boldsymbol{\theta}^{*}\right]\right) .
\end{aligned}
$$

That provides the estimate on the error e behavior and terminates the proof.

Remark. The condition on existence of the matrices $\mathbf{W}_{q}, q \in\{x, u, \theta\}$, looks like hard to satisfy; however, it can be easily fulfilled under certain structural restrictions imposed on $\mathbf{F}$. For example, this condition is always true for $\mathbf{W}_{q}=0$ and some $\tau_{q}$ and $\alpha_{q}$ such that $\tau_{q} \Delta_{q}^{\mathrm{T}} \Delta_{q} \leq \alpha_{q} \mathbf{I}_{g}, q \in\{x, u, \theta\}$ (introduction of $\mathbf{W}_{q} \neq 0$ may relax the conservatism of LMI). Additionally, the conditions for $\mathbf{W}_{q}, q \in\{x, u, \theta\}$, have the form of Lyapunov inequalities and for interval sets $\Upsilon_{q}$, their solutions can be obtained by a conventional LMI-based routine. Next, if for all $F_{j}, j=\overline{1, g}$, the partial derivatives are sign definite elementwise, then it is possible to find some $\mathbf{W}_{q}$ such that for all $\Delta_{q} \in \Upsilon_{q}$, the inequalities are true:

$$
\Delta_{q}^{\mathrm{T}} \mathbf{W}_{q}+\mathbf{W}_{q}^{\mathrm{T}} \Delta_{q} \geq 0, q \in\{x, u, \theta\},
$$

then the Theorem 1 condition holds for $\tau_{q}=\alpha_{q}=0$ (if the inequalities (5) are strict, then $\alpha_{q}=0$ only); in these cases, the theorem conditions are reduced to LMI checking.

\subsection{Performance Optimization}

The estimate derived in Theorem 1 gives some hints on possible performance optimization for the observer (2). For example, minimization of the value $\kappa / \nu$ improves overall accuracy of estimation. The value $\lambda_{\max }(\mathbf{P})$ regulates the rate of the system convergence. Additional minimization of the values $\lambda_{\nu}, \lambda_{\theta}$, and $\lambda_{d}$ allows to increase robustness margins of the estimation error dynamics with respect to corresponding variables. Simultaneous maximization of the value $\lambda_{f}$ ensures improvement of the sensitivity with respect to $\mathbf{f}$. The obtained expressions for these coefficients indicate that their parallel optimization is not possible 
and a trade-off has to be found. Since such optimization is based on an upper estimate tuning, it does not provide an optimal solution (the conversation is about a suboptimal one).

The above discussion on the coefficients $\lambda_{\nu}, \lambda_{\theta}, \lambda_{f}$, and $\lambda_{d}$ optimization reveals that it is rather hard to optimize robustness of the system with respect to all variables $\mathbf{v}, \boldsymbol{\theta}$, and $\mathbf{d}$ with simultaneous improvement of sensitivity with respect to the faults f. Additionally, such an adjustment needs application of the nonlinear optimization routine. In the following section, the focus will be on particular cases (robustness with respect to $\mathbf{v}$ or sensitivity to harmonic signals $\mathbf{f}$ ).

\section{MONOTONE SYSTEM APPROACH}

Another approach for stability analysis and performance optimization is based on the system (4) reduction to linear majorant and minorant systems using monotone system techniques, with posterior solution of the optimization problem for these linear simplified systems. To apply the monotone systems theory, rewrite Eq. (4):

$$
\dot{\mathbf{e}}=\tilde{\mathbf{A}}(t) \mathbf{e}+\mathbf{w}(t)
$$

where

$$
\begin{aligned}
\tilde{\mathbf{A}}(t)=\left(\mathbf{A}-\mathbf{L}_{1} \mathbf{C}\right)+\mathbf{G}\left[\Delta_{x}(t)\left(\mathbf{H}-\mathbf{L}_{2} \mathbf{C}\right)-\Delta_{u}(t) \mathbf{L}_{3} \mathbf{C}-\Delta_{\theta}(t) \mathbf{L}_{4} \mathbf{C}\right] & ; \\
\mathbf{w}(t)=-\left\{\mathbf{L}_{1}+\mathbf{G}\left[\Delta_{x}(t)\right.\right. & \left.\left.\mathbf{L}_{2}+\Delta_{u}(t) \mathbf{L}_{3}+\Delta_{\theta}(t) \mathbf{L}_{4}\right]\right\} \mathbf{d}(t) \\
& +\mathbf{S v}(t)+\mathbf{G}\left\{\Delta_{u}(t) \mathbf{f}(t)+\Delta_{\theta}(t)\left[\boldsymbol{\theta}-\boldsymbol{\theta}^{*}\right]\right\}
\end{aligned}
$$

Under Assumption 1, the signal $\mathbf{w}$ is bounded $(\|\mathbf{w}\|<+\infty))$ as well as the matrix function of time $\tilde{\mathbf{A}}$.

Assumption 2. Let the matrix $\left(\mathbf{A}-\mathbf{L}_{1} \mathbf{C}\right)+\mathbf{G}\left[\Delta_{x}\left(\mathbf{H}-\mathbf{L}_{2} \mathbf{C}\right)-\Delta_{u} \mathbf{L}_{3} \mathbf{C}-\Delta_{\theta} \mathbf{L}_{4} \mathbf{C}\right]$ be Metzler for all $\Delta_{x} \in \Upsilon_{x}, \Delta_{u} \in \Upsilon_{u}$, and $\Delta_{\theta} \in \Upsilon_{\theta}$, all elements of $\mathbf{C}$ have the same sign.

Assumption 2 means that the system (6) is monotone and the above mentioned theory can be applied to their analysis and optimization. This assumption can be relaxed assuming existence of a linear transformation $\mathbf{e}=\mathbf{X} \varepsilon$, such that in the new coordinates $\varepsilon$, the matrix $\mathbf{X}^{-1} \tilde{\mathbf{A}}(t) \mathbf{X}$ be Metzler for all $\Delta_{x} \in \Upsilon_{x}$, $\Delta_{u} \in \Upsilon_{u}$, and $\Delta_{\theta} \in \Upsilon_{\theta}$. This relaxation is technical and skipped here for brevity of presentation. The matrix $\mathbf{C}$ has positive elements in a conventional case $\mathbf{C}=\left[\begin{array}{llll}1 & 0 & \ldots & 0\end{array}\right]$; thus, this condition is also a question of coordinate transformation. 


\subsection{Stability Conditions}

Theorem 2. Let Assumptions 1 and 2 hold. Let the gains $\mathbf{L}_{i}, i=\overline{1,4}$ be chosen to satisfy the elementwise constraint

$$
\left(\mathbf{A}-\mathbf{L}_{1} \mathbf{C}\right)+\mathbf{G}\left[\Delta_{x}\left(\mathbf{H}-\mathbf{L}_{2} \mathbf{C}\right)-\Delta_{u} \mathbf{L}_{3} \mathbf{C}-\Delta_{\theta} \mathbf{L}_{4} \mathbf{C}\right] \leq \overline{\mathbf{A}}
$$

for all $\Delta_{x} \in \Upsilon_{x}, \Delta_{u} \in \Upsilon_{u}$, and $\Delta_{\theta} \in \Upsilon_{\theta}$, where $\overline{\mathbf{A}}=\left(\mathbf{A}-\mathbf{L}_{1} \mathbf{C}\right)+\mathbf{G}\left[\bar{\Delta}_{x}(\mathbf{H}\right.$ $\left.\left.-\mathbf{L}_{2} \mathbf{C}\right)-\bar{\Delta}_{u} \mathbf{L}_{3} \mathbf{C}-\bar{\Delta}_{\theta} \mathbf{L}_{4} \mathbf{C}\right]$ is Metzler and Hurwitz for some matrices $\bar{\Delta}_{k}$, $k \in\{x, u, \theta\}$.

Then, in (1) and (2), the estimation error e stays bounded for all $t \geq 0$.

P r o of. Introduce the following auxiliary dynamical systems (they will be used for analysis purposes only):

$$
\begin{aligned}
\dot{\mathbf{e}}^{r}=\overline{\mathbf{A}} \mathbf{e}^{r}+\mathbf{w}^{r}(t), r \in\{m, M\},-\infty<\mathbf{w}^{m}(t) \leq & \mathbf{w}^{M}(t)<+\infty, \\
\mathbf{w}^{m}(t) & \leq 0 \leq \mathbf{w}^{M}(t),
\end{aligned}
$$

for all $t \geq 0$, where $\mathbf{e}^{r} \in \mathbb{R}^{n}, r \in\{m, M\}$, and the initial conditions are $\mathbf{e}^{m}(0)$ $\leq \mathbf{e}(0) \leq \mathbf{e}^{M}(0), \mathbf{e}^{m}(0) \leq 0 \leq \mathbf{e}^{M}(0)$ (all vector inequalities are understood elementwise). Since the matrix $\overline{\mathbf{A}}$ is Hurwitz and $\left\|\mathbf{w}^{r}\right\|<+\infty, r \in\{m, M\}$, the variables $\mathbf{e}^{r}, r \in\{m, M\}$, are bounded for all $t \geq 0$. Moreover, $\mathbf{e}^{M}(t) \geq 0$, $\mathbf{e}^{m}(t) \leq 0$ for all $t \geq 0$ for Metzler matrix $\overline{\mathbf{A}}$ and sign definite initial conditions and input signals $\mathbf{w}^{r}, r \in\{m, M\}$. Define two relative errors $\varepsilon^{M}=\mathbf{e}^{M}-\mathbf{e}$ and $\varepsilon^{m}=\mathbf{e}-\mathbf{e}^{m}$, then

$$
\begin{array}{r}
\dot{\varepsilon}^{M}=\overline{\mathbf{A}} \mathbf{e}^{M}-\tilde{\mathbf{A}}(t) \mathbf{e}+\mathbf{w}^{M}(t)-\mathbf{w}(t) \\
=\tilde{\mathbf{A}}(t) \varepsilon^{M}+[\overline{\mathbf{A}}-\tilde{\mathbf{A}}(t)] \mathbf{e}^{M}+\left[\mathbf{w}^{M}(t)-\mathbf{w}(t)\right] ; \\
\dot{\boldsymbol{\varepsilon}}^{m}=\tilde{\mathbf{A}}(t) \mathbf{e}-\overline{\mathbf{A}} \mathbf{e}^{m}+\mathbf{w}(t)-\mathbf{w}^{m}(t) \\
=\tilde{\mathbf{A}}(t) \varepsilon^{m}+[\tilde{\mathbf{A}}(t)-\overline{\mathbf{A}}] \mathbf{e}^{m}+\left[\mathbf{w}(t)-\mathbf{w}^{m}(t)\right]
\end{array}
$$

By Assumption 2, the matrix $\tilde{\mathbf{A}}(t)$ is Metzler for all $t \geq 0$ and the signals $[\overline{\mathbf{A}}-\tilde{\mathbf{A}}(t)] \mathbf{e}^{M}+\left[\mathbf{w}^{M}(t)-\mathbf{w}(t)\right],[\tilde{\mathbf{A}}(t)-\overline{\mathbf{A}}] \mathbf{e}^{m}+\left[\mathbf{w}(t)-\mathbf{w}^{m}(t)\right]$ are elementwise positive for all $t \geq 0$; therefore, the variables $\varepsilon^{M}(t)$ and $\varepsilon^{m}(t)$ are also positive for all $t \geq 0$ since $\varepsilon^{M}(0) \geq 0$ and $\varepsilon^{m}(0) \geq 0$. Indeed, if there exists a coordinate $\varepsilon_{i}^{r}(t), i \in\{\overline{1, n}\}, r \in\{m, M\}$, approaching zero for some $t \geq 0$, then necessarily $\dot{\varepsilon}_{i}^{r}(t) \geq 0$ from the conditions above that prevents change of the sign. Thus, $\mathbf{e}^{m}(t) \leq \mathbf{e}(t) \leq \mathbf{e}^{M}$ for all $t \geq 0$ due to positivity of $\varepsilon^{M}(t)$ and $\varepsilon^{m}(t)$ that implies e boundedness.

It follows from Theorem 2 that under the monotonicity Assumption 2, the matrix inequalities from Theorem 1 can be replaced with some simple additive linear matrix constraints. The projection algorithm (3) becomes redundant in this case. 


\subsection{Performance Optimization}

To formulate the optimization criteria, the following definitions are needed. Let $\gamma: \mathbb{R}_{+} \rightarrow \mathbb{R}_{+}$be the stability margin gain (that is, a nonlinear counterpart of $H_{\infty}$ gain [24]) for the estimation error e with respect to the input $\mathbf{v}$, i. e.,

$$
\lim _{t \rightarrow+\infty}|\mathbf{e}(t)| \leq \gamma(\|\mathbf{v}\|)
$$

For any $\mathbf{f}(t)=\varepsilon \alpha \sin (\omega t), \varepsilon=[1 \ldots 1]^{\mathrm{T}} \in \mathbb{R}^{m}$ and some $\alpha>0, \omega>0$, let $\nu: \mathbb{R}_{+}^{2} \rightarrow \mathbb{R}_{+}$be the output frequency response map for (1) and (2) (see [25] for such function definition for the class of convergent systems; for generic case, such type of maps can be introduced using the theory of Cauchy gains and asymptotic amplitudes [26]), i.e.,

$$
\lim _{t \rightarrow+\infty}|\mathbf{C e}(t)| \leq \nu(\alpha, \omega) .
$$

Corollary 1. Let conditions of Theorem 2 hold, then

$$
\gamma(\|\mathbf{v}\|) \leq\left|\overline{\mathbf{A}}^{-1} \overline{\mathbf{S}}\|\mathbf{v}\|, \nu(\alpha, \omega) \leq \alpha \max _{r \in\{m, M\}}\right| W^{r}(i \omega) \mid
$$

where $\bar{S}_{i, j}=\left|S_{I, j}\right|, i=\overline{1, n}, j=\overline{1, \nu} ; W^{r}(s)=\mathbf{C}\left(\mathbf{I}_{n} s-\overline{\mathbf{A}}\right)^{-1} \mathbf{G}^{r}, \mathbf{G}^{M}$ $=\max \left\{\sup _{\Delta_{u} \in \Upsilon_{u}} \mathbf{G} \Delta_{u},-\inf _{\Delta_{u} \in \Upsilon_{u}} \mathbf{G} \Delta_{u}\right\}, \mathbf{G}^{m}=-\mathbf{G}^{M}$.

Proof. Systems (7) determine the asymptotic behavior for (1) and (2) (the estimation accuracy bounds) and the limit quality of transients. These upper and lower bounds can be exact in the cases when $\tilde{\mathbf{A}}(t) \rightarrow \overline{\mathbf{A}}$ and $\mathbf{w}(t) \rightarrow \mathbf{w}^{r}(t)$, $r \in\{m, M\}$. Systems (7) are linear, their robustness and sensitivity analysis is simple and numerically tractable. For a linear system (due to the superposition principle), its response to different inputs can be analyzed independently.

The input $\mathbf{w}$ depends on $\mathbf{v}$ in linear fashion with the constant gain $\mathbf{S}$, then in the signals $\mathbf{w}^{r}, r \in\{m, M\}$, this term can be taken into account as $\mathbf{S}^{r} \overline{\mathbf{v}}(t)$ where $S_{i, j}^{m}=-\left|S_{i, j}\right|, S_{i, j}^{M}=\left|S_{i, j}\right|, \bar{\nu}_{j}=\left|\nu_{j}\right|, i=\overline{1, n}, j=\overline{1, \nu}$. For linear systems $\dot{\mathbf{e}}^{r}$ $=\overline{\mathbf{A}} \mathbf{e}^{r}+\mathbf{S}^{r} \overline{\mathbf{v}}(t), r \in\{m, M\}$, one has $\lim _{t \rightarrow+\infty} \mathbf{e}^{r}(t) \leq\left|\overline{\mathbf{A}}^{-1} \mathbf{S}^{r}\right|\|\overline{\mathbf{v}}\| \leq\left|\overline{\mathbf{A}}^{-1} \mathbf{S}^{r}\right|\|\mathbf{v}\|$. Therefore, $\gamma(\|\mathbf{v}\|) \leq\left|\overline{\mathbf{A}}^{-1} \overline{\mathbf{S}}\right|\|\mathbf{v}\|, \overline{\mathbf{S}}=\mathbf{S}^{M}$.

The harmonic input $\mathbf{f}$ influence on the signals $\mathbf{w}^{r}, r \in\{m, M\}$, can be evaluated using the term $\mathbf{G}^{r} \overline{\mathbf{f}}(t), r \in\{m, M\}$, where

$$
\begin{gathered}
\mathbf{G}^{M}=\max \left\{\sup _{\Delta_{u} \in \Upsilon_{u}} \mathbf{G} \Delta_{u},-\inf _{\Delta_{u} \in \Upsilon_{u}} \mathbf{G} \Delta_{u}\right\} ; \\
\mathbf{G}^{m}=-\mathbf{G}^{M} ; \quad \bar{f}_{k}(t)=\alpha|\sin (\omega t)|, \quad k=\overline{1, m} .
\end{gathered}
$$

Then, the equations $\dot{\mathbf{e}}^{r}=\overline{\mathbf{A}} \mathbf{e}^{r}+\mathbf{G}^{r} \overline{\mathbf{f}}(t), r \in\{m, M\}$, can be analyzed. According to Assumption 2, the matrix $\mathbf{C}$ has all elements with the same sign; 
thus, $\mathbf{C e}^{m}(t) \leq \mathbf{C e}(t) \leq \mathbf{C} \mathbf{e}^{M}(t)$ for all $t \geq 0$ with positive elements of $\mathbf{C}$, the reverse sign inequalities are satisfied for the negative elements of $\mathbf{C}$. Therefore, in system (1), (2), the output Ce response on the harmonic fault input $\mathbf{f}$ can be estimated using the standard Bode magnitude plot:

$$
\nu(\alpha, \omega) \leq \alpha \max _{r \in\{m, M\}}\left|W^{r}(i \omega)\right|, W^{r}(s)=\mathbf{C}\left(\mathbf{I}_{n} s-\overline{\mathbf{A}}\right)^{-1} \mathbf{G}^{r}, \quad r \in\{m, M\} .
$$

This approach provides clear guidelines for performance optimization. In some cases, an analytical solution can be obtained for minimization/maximization of $\left|\overline{\mathbf{A}}^{-1} \mathbf{S}^{r}\right|$ and $\left|W^{r}(i \omega)\right|, r \in\{m, M\}$. However, Assumption 2 could be rather restrictive: first, it may fail in some applications; second, even being verified, the system with nonmonotone dynamics may have better performance.

\section{NUMERICAL EXAMPLE}

In this section, the ideas presented in this paper are illustrated through analytical design of a harmonic oscillatory failure case detector in electronic flight control system [20]. These faults may result in an unwanted control surface oscillation, generating unacceptably high loads or vibrations on the aircraft structure. The capability to detect robustly and as fast as possible these failures is very important because it has an impact on the structural design of the aircraft. In this paper, only failures located in the servocontrol loop of the moving surfaces is considered [20]. Habitually, such type of failure generates spurious sinusoidal signals (mainly, due to electronic components) propagating through the servocontrol loop, leading to control surface oscillation (see Fig. 1, where the structural scheme of servoloop is shown). The faulty components may be located inside the flight control computer, the analogue inputs/outputs, the position sensors, or the actuators. The flight control computer may also generate unwanted oscillations of the command current sent to the actuator servovalve. The fault signals are considered to be sinusoidal with amplitude and frequency uniformly distributed over the range $1-10 \mathrm{~Hz}$ (above $10 \mathrm{~Hz}$, the failure has no significant effects because of the low-pass nature of the actuator). The detection time is expressed in period numbers, thus depending on the failure frequency, the time permissible for detection is varying.

The following actuator model is considered [20]:

$$
\left.\begin{array}{rl}
\dot{x}(t) & =\varphi\left[y_{f}(t)-x(t)+u(t)+f(t)\right]+\nu(t) ; \\
y_{f}(t) & =W_{f}(p)[x(t)-u(t)-f(t)] ; \\
y(t) & =x(t)+d(t)
\end{array}\right\}
$$


(1) OFC sources

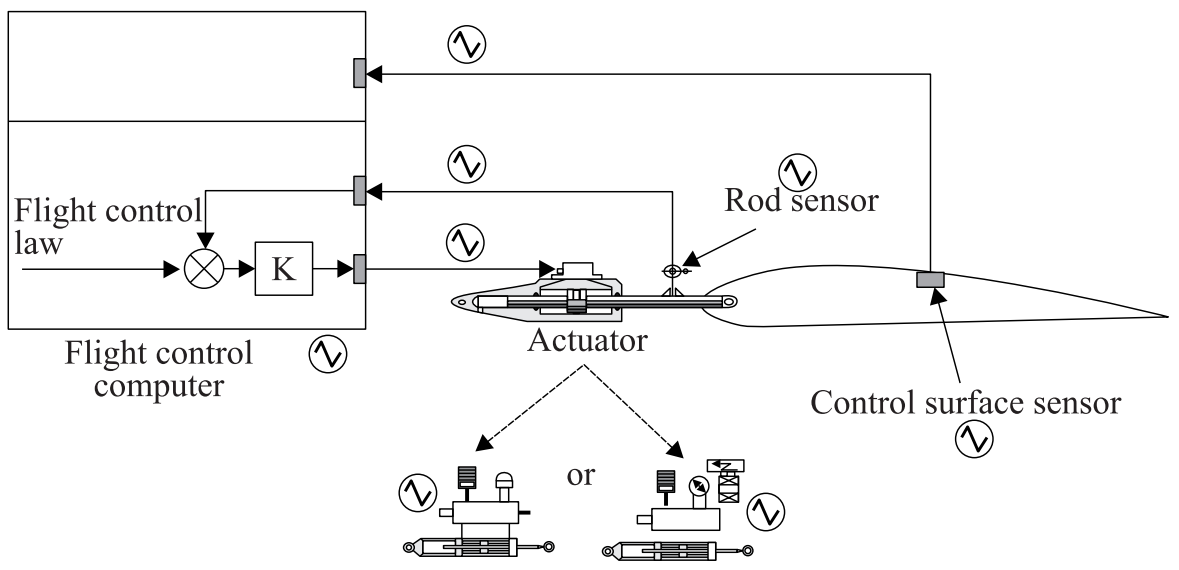

Hydraulic servocontrol Electrohydraulic actuator

Figure 1 Structural scheme of the actuator servoloop
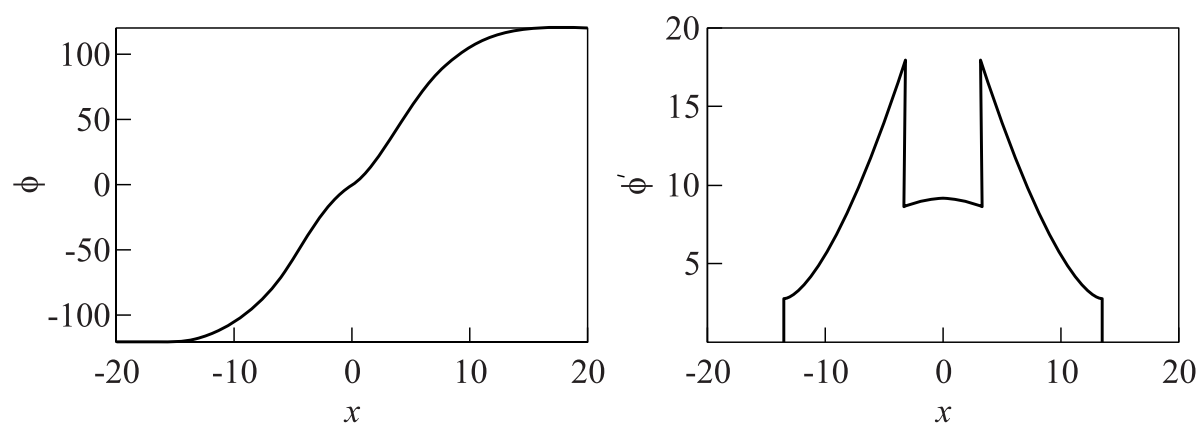

Figure 2 The actuator nonlinearity

where $x \in \mathbb{R}$ is the actuator rod position; $u \in \mathbb{R}$ is the control signal; $y \in \mathbb{R}$ is the available measurement output; $f \in \mathbb{R}$ is the sinusoidal fault; $\nu$ and $d$ are the disturbances as before; $y_{f}$ is the output of a filter; $p$ is stated for the time differentiation operator; and $W_{f}(p)$ is the filter transfer function. The function $\varphi$ and its derivative are given in Fig. 2; for simplicity, a high pass filter is considered in this work: $W_{f}(p)=-T p /(T p+1), T=10$.

The model (8) can be presented in the form (1) introducing the following functions and matrices: 


$$
\begin{aligned}
& 3 \mathbf{A}=\frac{1}{T}\left[\begin{array}{cc}
0 & 0 \\
1 & -1
\end{array}\right] ; \quad \mathbf{G}=\left[\begin{array}{cc}
1 & 0 \\
0 & T^{-1}
\end{array}\right] ; \quad \mathbf{F}(h, u)=\left[\begin{array}{c}
\varphi(h+u) \\
u
\end{array}\right] \\
& \mathbf{S}=\left[\begin{array}{l}
1 \\
0
\end{array}\right] ; \quad \mathbf{H}=\left[\begin{array}{c}
-1 \\
1
\end{array}\right]^{\mathrm{T}} ; \quad \mathbf{C}=\left[\begin{array}{l}
1 \\
0
\end{array}\right]^{\mathrm{T}} \text {. }
\end{aligned}
$$

Assume that the sets required in Assumption 1 are given. Since the system (8) has one nonlinearity only (the condition (5) is satisfied), after some transformations the observer (2) can be presented as follows:

$$
\left.\begin{array}{l}
\dot{e}_{1}=\Delta(t)\left[e_{2}-e_{1}+f-\left(L_{2}+L_{3}\right)\left(e_{1}+d\right)\right]-L_{11}\left(e_{1}+d\right)+\nu ; \\
\dot{e}_{2}=T^{-1}\left(e_{1}-f-e_{2}\right)-\left(L_{12}-T^{-1} L_{3}\right)\left(e_{1}+d\right)
\end{array}\right\}
$$

where $\Delta=\varphi^{\prime}\left(\eta^{h}\right)$ and, according to Fig. $2,0 \leq \Delta(t) \leq \dot{\varphi}_{\max }=18.11$ for all $t \geq 0$. For the matrix $\mathbf{P}=\mathbf{I}_{2}$, the estimate from Theorem 1 on the error dynamics takes the form:

$$
\begin{aligned}
|\mathbf{e}(t)| \leq|\mathbf{e}(0)| e^{-0.125 t / T} & +2 \sqrt{2}\left[\sqrt{\dot{\varphi}_{\max }+\frac{4}{T}}\|f\|+\|\nu\|\right. \\
& \left.+\left(\left|L_{11}+\dot{\varphi}_{\max }\left\{L_{2}+L_{3}\right\}\right|+2\left(L_{12}-T^{-1} L_{3}\right)\right)\|d\|\right] .
\end{aligned}
$$

Thus, the gain before $\|d\|$ is the only one available for optimization using the observer gains tuning.

To apply Theorem 2, note that the system (9) is monotonous while $T^{-1}\left(1-L_{3}\right) \geq L_{12}$. Under this constraint,

$$
\tilde{\mathbf{A}}(t)=\left[\begin{array}{cc}
-L_{11}-\left(1+L_{2}+L_{3}\right) \Delta(t) & \Delta(t) \\
T^{-1}-\left(L_{12}-T^{-1} L_{3}\right) & -T^{-1}
\end{array}\right]
$$

is a Metzler matrix for any $0 \leq \Delta(t) \leq \dot{\varphi}_{\max }$. Then,

$$
\overline{\mathbf{A}}=\left[\begin{array}{cc}
-L_{11} & \dot{\varphi}_{\max } \\
T^{-1}-\left(L_{12}-T^{-1} L_{3}\right) & -T^{-1}
\end{array}\right] ; \mathbf{G}^{m}=\left[\begin{array}{c}
-\dot{\varphi}_{\max } \\
T^{-1}
\end{array}\right] ; \mathbf{G}^{M}=\left[\begin{array}{c}
\dot{\varphi}_{\max } \\
T^{-1}
\end{array}\right] ;
$$

and $\overline{\mathbf{A}}$ is Metzler. The matrix $\overline{\mathbf{A}}$ is Hurwitz under proper choice of the gains $L_{11}, L_{12}$, and $L_{3}$. The analytical formulas for gains to be optimized are as follows: 


$$
\begin{aligned}
\left|\overline{\mathbf{A}}^{-1} \overline{\mathbf{S}}\right| & =\gamma\left(M_{1}, M_{2}\right)=\frac{\sqrt{1+M_{2}^{2}}}{\left|M_{1}-\dot{\varphi}_{\max } M_{2}\right|} ; \\
\left|W^{M}(i \omega)\right| & =\beta\left(\omega, M_{1}, M_{2}\right)=\dot{\varphi}_{\max } \frac{\sqrt{4+T^{2} \omega^{2}}}{D\left(\omega, M_{1}, M_{2}\right)} ; \\
\left|W^{m}(i \omega)\right| & =\frac{\dot{\varphi}_{\max } T \omega}{D\left(\omega, M_{1}, M_{2}\right)} ; \\
D\left(\omega, M_{1}, M_{2}\right) & =\sqrt{\omega^{2}\left(1+M_{1} T\right)^{2}+\left(M_{1}-T \omega^{2}-\dot{\varphi}_{\max } M_{2}\right)^{2}}
\end{aligned}
$$

where $M_{1}=L_{11}$ and $M_{2}=1-L_{12}+L_{3}$ are new tuning parameters. Note that

$$
\max _{r \in\{m, M\}}\left|W^{r}(i \omega)\right|=\left|W^{M}(i \omega)\right|=\beta\left(\omega, M_{1}, M_{2}\right) .
$$

The values of $\gamma\left(M_{1}, M_{2}\right)$ and $\beta\left(\omega, M_{1}, M_{2}\right)$ could be optimized numerically for new set of planar parameters $M_{1}$ and $M_{2}$ taking in the mind the stability of matrix $\overline{\mathbf{A}}$ and the monotonicity constraint $M_{2} \geq 0$. The following functional has been chosen for minimization:

$$
J\left(M_{1}, M_{2}\right)=\kappa \gamma\left(M_{1}, M_{2}\right)+\frac{1-\kappa}{\sum_{\omega \in \Omega_{f}} \beta\left(\omega, M_{1}, M_{2}\right)}
$$

where $\Omega_{f}=\{1,2,3,4,5,7\}$ is the set of the fault most important frequencies that could happen; and $0 \leq \kappa \leq 1$ is the weighting parameter. The contour plot of this functional for $\kappa=0.3$ is shown in Fig. 3, where the left low uncolored corner corresponds to the cases with unstable matrix $\overline{\mathbf{A}}$. The values of the observer gains $L_{11}=12, L_{12}=0.1$, $L_{2}=1$, and $L_{3}=0.08$ provide the minimum of this functional.

These gains form a suboptimal solution in the sense of the observer (2) response optimization. An example of the residual signal $e_{1}$ obtained for these observer gains is shown in Fig. 4 where for simulation, it was

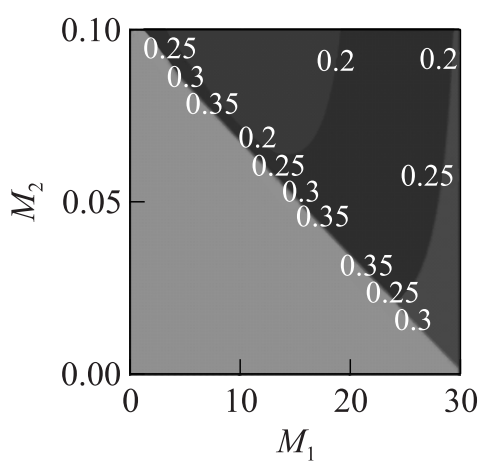

Figure 3 Performance functional values used:

$u=\sin (t)+\sin (0.2 t)+\cos (2 t) ; \nu(t)=\sin (10 t) ; f(t)= \begin{cases}0, & \text { if } t \leq 10 \\ 0.2 \sin (3 t), & \text { if } t>10\end{cases}$ 


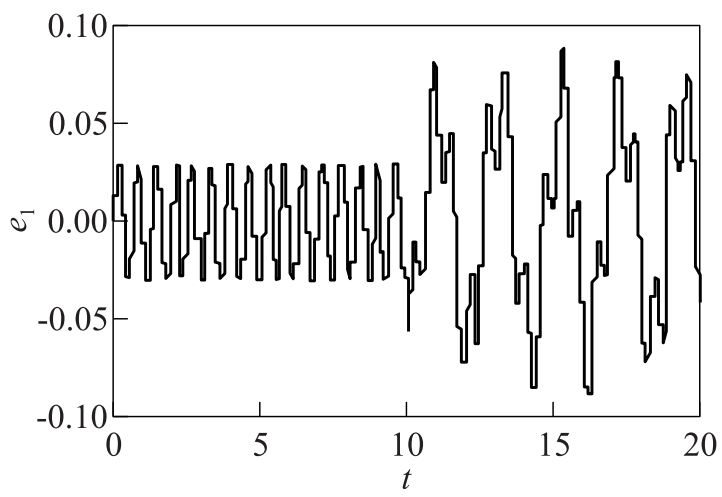

Figure 4 The results of simulation

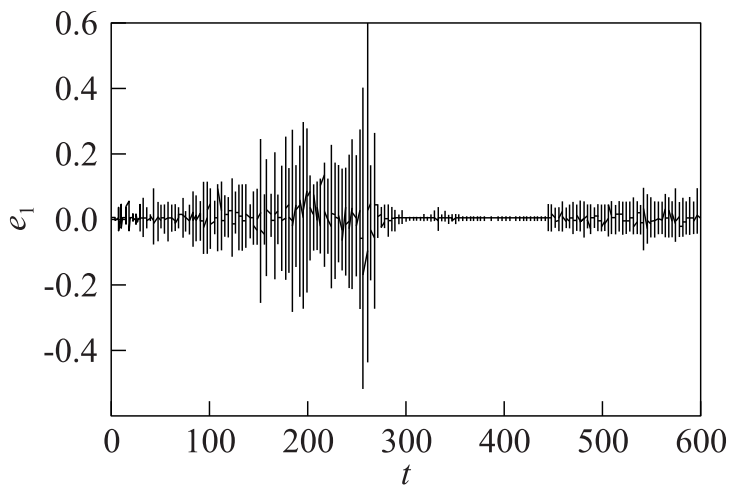

Figure 5 The output residual in the ADDSAFE benchmark

This technique has been successfully verified on the OFC detection problem in the European FP7 ADDSAFE project, the result of this algorithm operation in the ADDSAFE benchmark is shown in Fig. 5.

For nonlinear systems, any type of optimization is a complex issue, even choice of an optimizing functional corresponding to the posed performance goal is a hard problem.

In this example, for instance, application of Theorem 1 does not provide a hint how to evaluate the output estimation error sensitivity with respect to harmonic faults. Theorem 2 provides one with a functional in a systematic way that is a big advantage of the presented approach. 


\section{CONCLUDING REMARKS}

The problem of nonlinear observer design for fault detection with optimized performance is studied. It is assumed that the plant model contains unknown parameters and it is subjected by external disturbances and faults. Two approaches for observer design are presented. The first one is based on solution of LMIs, its novelty consists in introduction of additional observer gains in the conventional routine for LMI-based observer design. The additional observer gains may be used for performance optimization. The second method uses monotonicity assumption on the estimation error dynamics, it introduces a new tool to design nonlinear observers. An advantage of the second approach is that it gives a simple technique to tune the observer gains in order to optimize the fault detection performance and robustness. Efficiency of the proposed approach is demonstrated through the oscillatory failure case in aircraft surface servoloops.

\section{ACKNOWLEDGMENTS}

The authors are grateful for the provision of an EU FP7 grant ADDSAFE (FP7233815) which funded this work.

\section{REFERENCES}

1. Chen, J., and R. J. Patton. 1999. Robust model-based fault diagnosis for dynamic systems. Kluwer Academic Publs.

2. Ding, S.X. 2008. Model-based fault diagnosis techniques. Design schemes, algorithms, and tools. Heidelberg, Berlin: Springer.

3. Bortoff, S. A., and A.F. Lynch. 1995. Synthesis of optimal nonlinear observers. 34th Conference on Decision and Control Proceedings. 95-100.

4. Ding, S. X., T. Jeinsch, P. M. Frank, and E. L. Ding. 2000. A unified approach to the optimization of fault detection systems. Int. J. Adaptive Control Signal Proc. 14:725-45.

5. Wang, J., G. Yang, and J. Liu. 2007. An LMI approach to $H_{-}$index and mixed $H_{-} / H_{1}$ fault detection observer design. Automatica 43:1656-65.

6. Casavola, A., D. Famularo, and G. Franze. 2008. Robust fault detection of uncertain linear systems via quasi-LMIs. Automatica 44:289-95.

7. Li, X., and K. Zhou. 2009. A time domain approach to robust fault detection of linear time-varying systems. Automatica 45:94-102.

8. Zhong, M., S.X. Ding, and E. L. Ding. 2010. Optimal fault detection for linear discrete time-varying systems. Automatica 46:1395-400. 
9. Leondes, C. T., and J. F. Yocum. 1975. Optimal observers for continuous time linear stochastic systems. Automatica 11(1):61-73.

10. Simon, D. 2006. Optimal state estimation: Kalman, $H$ infinity, and nonlinear approaches. Wiley-Interscience.

11. Poznyak, A., A. Nazin, and D. Murano. 2004. Observer matrix gain optimization for stochastic continuous time nonlinear systems. Systems Control Lett. 52(5):377-85.

12. Abbaszadeh, M., and H. J. Marquez. 2008. Robust $H_{\infty}$ observer design for sampleddata Lipschitz nonlinear systems with exact and Euler approximate models. Automatica 44(3):799-806.

13. Moisana, M., O. Bernard, and J.-L. Gouzé. 2009. Near optimal interval observers bundle for uncertain bioreactors. Automatica 45(1):291-95.

14. Nijmeijer, H., and T. I. Fossen. 1999. New directions in nonlinear observer design. London, U.K.: Springer-Verlag.

15. Besançon, G., ed. 2007. Nonlinear observers and applications. Lecture notes in control and information science ser. Berlin: Springer Verlag. 363.

16. Smith, H. L. 1995. Monotone dynamical systems: An introduction to the theory of competitive and cooperative systems. Surveys and monographs. Providence: AMS. 41.

17. Lee, L. H. 1997. Identification and robust control of linear parameter-varying systems. PhD Thesis. Berkeley, CA: University of California at Berkeley.

18. Bokor, J., and G. Balas. 2004, Detection filter design for LPV systems - ageometric approach. Automatica 40:511-18.

19. Raïssi, T., G. Videau, and A. Zolghadri. 2010. Interval observers design for consistency checks of nonlinear continuous-time systems. Automatica 46(3):518-27.

20. Goupil, P. 2010. Oscillatory failure case detection in the A380 electrical flight control system by analytical redundancy. Control Eng. Practice 18:1110-19.

21. Arcak, M., and P. Kokotović. 2001. Nonlinear observers: A circle criterion design and robustness analysis. Automatica 37:1923-30.

22. Alcorta-Garcia, E., A. Zolghadri, and P. Goupil. 2011. A nonlinear observer-based strategy for aircraft oscillatory failure detection: A380 case study. IEEE Trans. Aerospace Electronic Syst. 47(4):2792-806.

23. Pomet, J. B., and L. Praly. 1992. Adaptive nonlinear regulation: Estimation from the Lyapunov equation. IEEE Trans. Automatic Control AC-37:729-40.

24. Sontag, E. D. 2007. Input to state stability: Basic concepts and results. In: Nonlinear and optimal control theory. Eds. P. Nistri and G. Stefani. Berlin: SpringerVerlag. 163-220.

25. Pavlov, A. V., N. van de Wouw, and H. Nijmeijer. 2007. Frequency response functions for nonlinear convergent systems. IEEE Trans. Automatic Control 52:115965 .

26. Sontag, E. D. 2002. Asymptotic amplitudes and cauchy gains: A small-gain principle and an application to inhibitory biological feedback. Systems Control Lett. 47:167-79. 